\title{
Mathematical Model of Hepatitis B Transmission in Ondo State, Nigeria
}

\author{
Longe I. O. ${ }^{1} \quad$ Adeniran A. O. ${ }^{1 *} \quad$ Longe B. E. ${ }^{2}$ \\ 1.Department of Statistics, Federal Polytechnic, Ile-Oluji, Nigeria \\ 2. Department of Health Promotion and Education, University of Ibadan, Ibadan, Nigeria
}

\begin{abstract}
Hepatitis B is one of the viral diseases that is treating the global health, in this study, a classical SIR model for Hepatitis B transmission is considered. Based on the available data from the Ondo State Ministry of Health, associate parameters are estimated and the fitted model is shown by appropriate simulations. The basis reproduction number is obtained and other sensitivity analysis carried out. The result of the study shows that Hepatitis B can be eradicated if effort is geared at vaccinating susceptible individual to make rate of infection low and recovery rate high.
\end{abstract}

Keywords: HBV, Epidemiology, Disease, Compartment, Dynamics, model.

DOI: $10.7176 / \mathrm{MTM} / 11-4-01$

Publication date:September $30^{\text {th }} 2021$

\section{Introduction}

Hepatitis B (HB) is a life-threatening liver infection caused by Hepatitis B virus (HBV). It is an infectious disease transmitted by contacting the blood or body fluids of an infected person, it infection can cause acute chronic illness or liver cancer. World Health Organization (WHO) reported that people living with Hepatitis B Virus (HBV) are over 257 million. A lot of researchers have paid great attention to study of Hepatitis B viruses (HBV) (Su, Shou, Dorman, \& Jones (2009)) because it is a deadly disease and a life-threatening virus.

The vaccine to curtail Hepatitis B virus (HBV) has been made available since 1982, despite this, there is still and increase in the transmission and its spread. Mathematical model has been used to curtail its spread and dynamics. Mn, Su, \& Kuang (2008) proposed an infectious model whose compartment consist of uninfected target cells, infected cells and the density of the virus. Meltem (2019) discussed Hepatitis B infection as a disease that is threatening the global health in Turkey, a classical SIR model for Hepatitis B virus transmission is proposed and analyzed with respect to available data of Turkey Ministry of Health, associated parameters are estimate and fitted the basic reproductive number is obtained using the estimated parameters.

Motivated by the above-mentioned work in literature, we used an infectious disease model to understand the dynamic and prevalence of (HBV). In this paper, we present the model formulation of the transmission dynamic (Dynamic behavior of the model including its equilibria and stabilities) of HBV in Ondo State which is nicknamed sunshine state, a state located at the southwestern of Nigeria, created in February 1976 from the former western state, is the 18th largest state in the country with the population of over Three million $(3,000,000)$, which is predominantly Yoruba.

\section{Literature Review}

Chronister et. al. 1992, was of the opinion that Hepatitis B eye care practitioners are in the high-risk category for contracting and transmitting this serious viral infection. In other words, Hepatitis B can be said to be in the class of serious viral infections. He went further to opine that being present in all the bodily fluids, including tears, it is easily transmittable with high degree of infectiousness than Human Immunodeficiency Virus (HIV)

WHO 2015, says Hepatitis B Virus Infection is highly prevalent worldwide, with a disproportionately high burden in low and middle income countries. As much as this virus can be prevented by vaccines, the lack of awareness as heightened the spread, even in high income countries. Clinical Microbiology Reviews, 2020, statistically says that approximately 250 million people remain infected with Hepatitis B, which forms one of the major causes of liver cancer and overall mortality globally. In their opinion, despite the availability of vaccines and suppressive viral medications since 1998, linkage to care is still very poor, even in the USA, Western Europe and Japan.

Man-Fung et al, 2018, describes Hepatitis B as a hepatotropic virus that can establish a persistent and chronic infection in Humans through immune anergy. Most researchers, owe the wide spread of Hepatitis Bto poor vaccination rate, lack of awareness of infection status. Emenchebe et al, 2010, describes Hepatitis B as a pandemic and chronic infection which may lead to chronic liver diseases, which are often lethal. The researchers went further to opine that HBV transmission in Nigeria, occurs mainly during childhood, and other factors like sexual promiscuity, blood transmission, etc encourages the spread throughout the Country. Nishant Tripathi 2021 , in agreeing with some researchers on the transmission mode and description of Hepatitis B

Mark Geier, 2003, reiterates that even though Hepatitis B is a chronic disease, vaccines have formulated to 
combat the life-threatening disease. He posits that medical societies have generally that the recombinant hepatitis B vaccine- a highly purified, genetically engineered, single antigen vaccine- is a safe vaccine. Also, he was of the opinion that the components of the vaccine, which contains yeast, aluminum, thimerosal and hepatitis B surface antigen epitopes, may result in hepatitis B vaccine being associated with autoimmune diseases among susceptible adult vaccine recipients. In the researcher's opinion, the benefits of the vaccine far outweigh its risk.

From a different point of view, the National Academies Press (US) 2002,

US National Library of Medicine says that the HBV Vaccine is to prevent Hepatitis B, and posits that the vaccine is usually given in 2,3 , or 4 shots to infants, adolescent, or unvaccinated adults in different categories.

Scott Frothingham, 2018, says the best way to prevent the Hepatitis B infection is to get the vaccine. While explaining that the first and second dose are given one month apart, and the last dose taken after six months from the second dose. Seen as generally safe, the researchers suggest that people with history of hypersensitivity to yeast or those going through serious illness are not advised to take the vaccine. April Khan, 2018, statistically says that the Centers for Diseases Control and Prevention state that around 3000 people in the United States die each year from complications caused by Hepatitis B and 1.4 million people in America have chronic hepatitis B.

April Khan, 2018, posits that Acute Hepatitis B does not usually require treatment, because most people would overcome an acute infection on their own, while rest and hydration helps in recovering. In the researcher's opinion, Chronic Hepatitis B is treated with anti-retroviral medications.

Ding-Shinn Cheng, 2009, sees Hepatitis B as a Global health issue, with about 360 million infected people. The researcher says the infection can now be treated by antiviral or interferon and the transmission route can be interrupted, but ensured to say that the most effective treatment is to immune all susceptible people, especially young children, with safe and efficacious vaccines. The combined efforts of vaccination, effective treatment and interruption of transmission make elimination of the infection plausible and may lead to the eradication of the virus (Ding-Shinn Cheng, 2009), meanwhile, while partially agreeing with the above researcher, WHO, 2020 says there is no specific treatment for acute Hepatitis B, and as such the care is maintaining comfort and nutritional balance, replacement of fluids, and most important is the avoidance of unnecessary medications. Chronic Hepatitis B can be treated with medicines, including oral antiviral agents. This treatment can slow the progression of cirrhosis, incidence of liver cancer and improve long term survival (WHO, 2020)

In a bid to understand the dynamics of various diseases and infections and to study the spread of diseases, it is pertinent to use a viable mathematical model, which was used in different modes by Kermack and McKendrick in understanding the dynamics of various diseases.Zahura Khatun, 2020, says mathematical modeling is used to observe and understand the spreading of infections. MeltemGolgeli, 2019, posits that the Kermack and McKendrick Modelling suggest three compartments, i. e. the Susceptible, the Infective and the Recovered ones. In the study of epidemics, research shows the challenges faced in finding the causal factor, which may show the frequency of the disease. It was in view of finding the frequency and obtaining more insight the causal factor and effects of spread of infections that led to the Kermack and McKendrick.

Isaac Kwasi et al, 2014 used the SIR model, which is a modification of the Kermack and McKendrick model, with the use of the three compartments used under the Kermack and McKendrick Model.The assumption of the model is that the disease spreads in a closed environment; that is there is no emigration or immigration, and neither birth nor death in the population, so that the total population remains a constant for all $t$ Isaac Kwasi et al, 2014.

Dongmei Chen, 2014, Considers SIR model as the simplest of all population based mathematical models, and describes SIR model as formerly known as Kemack and McKendrick Model in 1927. The researcher posits that the model was proposed to explain the rise and fall in the number of infected patients in epidemics, such as the plague; London 1665-1666, and Cholera; London 1865. The researcher considers this model and intuitivebased. The use of this model shows that at any given time, the susceptible may become infected, while those who are infected join the recovered segment.

Iman Rahimi, et al, 2020, observed that the SIR Model was used for COVID-19 Epidemic, as themost effective, whereas the E factor was added to for the segment of individuals that have been infected but are Asymptomatic. The result shows that the accuracy of the Model is determined by the Federal and State level policies. In agreement with some other researchers, Lu Tang, et al, 2020, posits that SIR model can accommodate more compartments, such as the E factor for COVID-19 Epidemic, which takes into account the incubation period. The researcher suggests that the advantage of this model is that the model parameters, including those in the mechanistic model, can be estimated.

Ian Cooper, et al, agrees with other researchers on the usage of the SIR Model in providing a theoretical framework to study the spread of epidemics, as well as the compartments involved, as to the suspected, infected and recovered population, with the result that the population is not defined or keep at a constant number, however, the researcher, on the contrary, posits that using this model, the number can increase in surge periods, using the COVID-19 era as an example. 


\section{Model Formulation}

The modeling approach of Kermach and Mc Kendric was used, we assumed the model have three different compartment the Susceptible $(\mathrm{S}(\mathrm{t}))$; who are individuals that have the potential of getting infected, the Infective $(\mathrm{I}(\mathrm{t}))$ are individuals who are infected and able to transmit $\mathrm{HBV}$; Recovered $(\mathrm{R}(\mathrm{t}))$ are individuals who have recovered and also gained immunity.

\section{Model Assumptions}

The model is based on the following assumptions

$\$$ The population is heterogeneous, hence the individual that make up the population can be grouped into compartments according to epidemiological state.

* The population size in the compartments is differentiable with respect to time and the epidemic is deterministic

* People in compartments have equal death rate

\# No immigrants and emigrants, the only entry point is through birth, while the only exit is though death from natural/other causes or death from HBV related causes.

\section{Model Diagram}

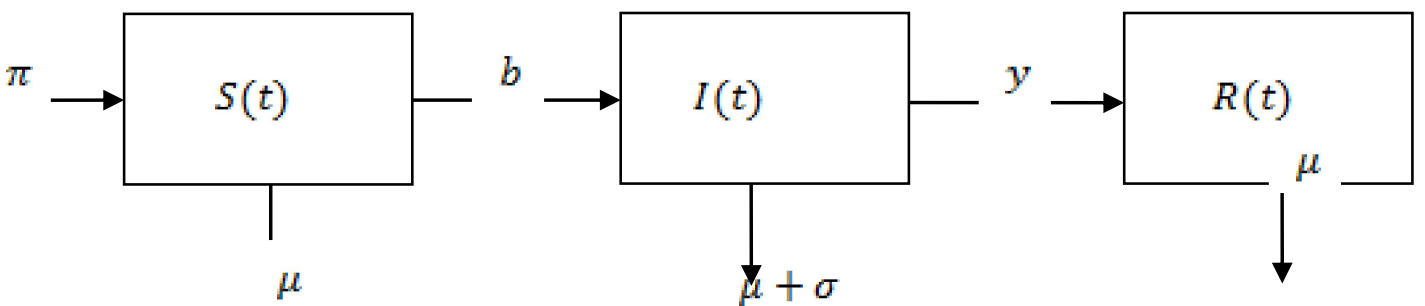

Figure 1: Schematic Diagram of HBV Transmission

\section{Governing equations}

Considering the model assumptions and diagrams, the model describing HB transmission is formulated as a system of Ordinary Differential Equations (ODE). The dependent variables are $S(t), I(t)$ and $R(t)$. The equations reads

$$
\begin{gathered}
\quad \frac{d S(t)}{d t}=\pi-b S(t) I(t)-\mu S(t), \\
\frac{d I(t)}{d t}=b S(t) I(t)-(y+\mu+\sigma) I(t), \\
\frac{d R(t)}{d t}=y I(t)-\mu R(t) . \\
\text { With initial condition } \mathrm{S}(0)=\mathrm{S}(0) \quad \mathrm{I}(0)=\mathrm{I}_{0}>0 \text { and } \mathrm{R}(0)=0
\end{gathered}
$$

Table 1: Parameters description

\begin{tabular}{ccc} 
Parameter & Description & Unit \\
$\pi$ & Constant Birth Rate & Number/Unit time \\
$\boldsymbol{\mu}$ & Death Rate & Rate/Unit time \\
\cline { 2 - 3 } $\mathrm{B}$ & Transmission Rate & Rate/Unit time \\
\hline $\boldsymbol{r}$ & Disease Death rate & Rate/Unit time \\
$\mathrm{Y}$ & Recovered Rate & Rate/Unit time
\end{tabular}

\section{Model Analysis}

With respect to hypothesis of conservation of population, we have

$$
N=\mathrm{S}(\mathrm{t})+\mathrm{I}(\mathrm{t})+\mathrm{R}(\mathrm{t})
$$

Where $N$ is the constant population (Murray (1993)), parameter $\pi$ and $\mu$ represent a constant birth rate and a constant death rate respectively, parameter $b$ represents how fast a susceptible individual becomes infected with $\mathrm{HBV}$, parameter $\mathrm{y}$ is the recovery rate whichcontrol the rate at which recovered individuals become reinfected by $\mathrm{HBV}$, Parameter $\sigma$ is the death rate as a result of the disease, all parameter are assumed to be positive,equation (3.1) can be reduced since $\mathrm{R}$ is decoupled with the first two equations and obtained from equation (3.1). We seek the equilibria using the classical linearization techniques,

$$
\frac{d S(t)}{d t}=\frac{d I(t)}{d t}
$$

We have

$$
\begin{aligned}
& =0 \\
& \pi-b S(t) I(t)-\mu S(t)=0
\end{aligned}
$$




$$
b S(t) I(t)-(y+\mu+\sigma) I(t)=0
$$

The disease free state is obtained when $\mathrm{I}(\mathrm{t})=0$, Thus the equilibrium for disease free state is given as

$$
=\left(\frac{\pi}{\mu}, 0\right)
$$

Similarly, when $I(t) \neq 0$, we obtained the endemic state for model (3.1) as

$$
\begin{aligned}
E^{* *}\left(S^{* *}, I^{* *}\right) & =\left(\frac{y+\mu+\sigma}{b}, \frac{b \pi-\mu^{2}-\mu \sigma-\mu y}{b(y+\mu+\sigma)}\right. \\
& \left.-\frac{y\left(\pi b-\mu^{2}-\mu \sigma-\mu y\right.}{b(y+\mu+\sigma)}\right)
\end{aligned}
$$

On evaluating the Jacobian of the equation (3.1), we have

$$
I=\left(\begin{array}{ccc}
-\mathrm{bI}(\mathrm{t})-\mu & b S(t) & 0 \\
b I(t) & b S(t)-(\mu+\sigma+y) & 0 \\
y I(t) & y & -\mu
\end{array}\right)
$$

For stability analysis, we find the exact value of the Jacobian matrix at equilibria in Equation (3.5) at the disease free state.

$$
J_{e}=\left(\begin{array}{ccc}
-\mu & \mathrm{b}\left(\frac{\pi}{\mu}\right) & 0 \\
0 & \mathrm{~b}\left(\frac{\pi}{\mu}\right)-(\mu+\sigma+y) & 0 \\
0 & y & -\mu
\end{array}\right)
$$

The Eigen value $\lambda_{1}=-\mu, \lambda_{2}=-\mu$ and $\lambda_{3}=\frac{\pi b-\mu^{2}-\mu \sigma-\mu y}{\mu}$ are obtained from the characteristic equation given by $\operatorname{det}\left(J_{e}-\lambda I\right)=0$, where $\lambda$ is the characteristics matrix of the equation.

Theorem 2.1: The necessary and sufficient condition for the asymptotic stability of disease free equilibria is that the real parts of all Eigen values be negative (hedder, 2013)

Therefore, $\lambda_{1}, \lambda_{2}=-\mu<0$ and the stability of the disease free equilibrium depends on the parameters, $\pi, b, y, \sigma$ and $\mu$ if $\lambda_{3}=\frac{\pi b-\mu^{2}-\mu \sigma-\mu y}{\mu}<0$ then all the eigenvalues becomes negative.

Assuming that in the beginning of the epidemic $\mathrm{S}(0)=S_{0}>0, \mathrm{I}(0)=I_{0}>0$ and $\mathrm{R}(0)=0$ are given, we define the basic reproduction rate

$$
\mathrm{R}=\frac{b}{y+\mu+a}
$$

Which determines the expected number of the secondary cases generated from an infected individual, thus the old threshold value for the behavior of the epidemic spreads if $\mathrm{R}>1$ and die out if $\mathrm{R}<1$ (James and Sleemen (2003))

\section{Application of the Model to the data of Hepatitis B in Ondo State, Nigeria}

We collected Data for this study from the Ondo State Ministry of Health,Akure, Ondo State, the collected was analyzed and used to validate the epidemiology model.

Figure 1. Morbidity, OPD and Mortality for seven (7) consecutive years

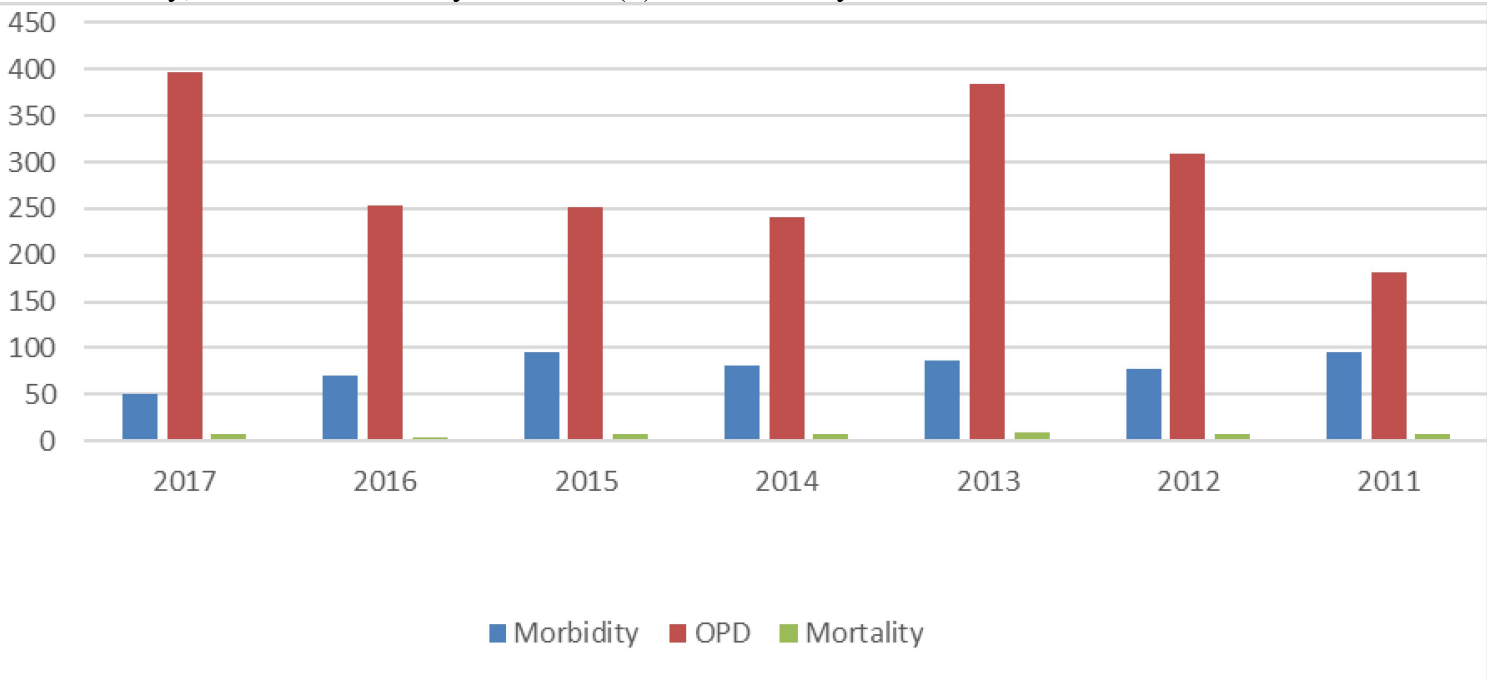


Figure 1 above shows that year 2015 has the highest morbidity rate, while 2017 has the lowest. Also year 2017 has the highest OPD while 2011 has the lowest. Likewise for Mortality 2013 has the highest and 2016 recorded the least death.

The SIR model in equation 2.1 was solved numerically using Runge-Kutta of order four embedded inside Maple 17.

Table 2: Model parameter values

$\begin{array}{cc}\text { Parameter } & \text { Unit } \\ \pi & 6.0 \\ \boldsymbol{\mu} & 0.092 \\ \mathrm{~b} & .275 \\ \mathrm{y} & 0.908\end{array}$

These parameters above are used in the plotting of the graphs. The parameters determines whether the disease will persist or will be eradicated in due time. Figure 2, 3 and 4 shows the Susceptible, Infected and Recovered Individuals.

Figure 2 shows that those likely to be affected will be constant in due time as knowledge and perception of the disease increase among residents of Ondo State.

Figure 3 shows the infected among the resident of Ondo State, from the curve, it can be deduced the rate of infected will reduce drastically as time goes on.

Figure 4 shows the recovery graph, which signifies high recovery rate among those infected with the disease.

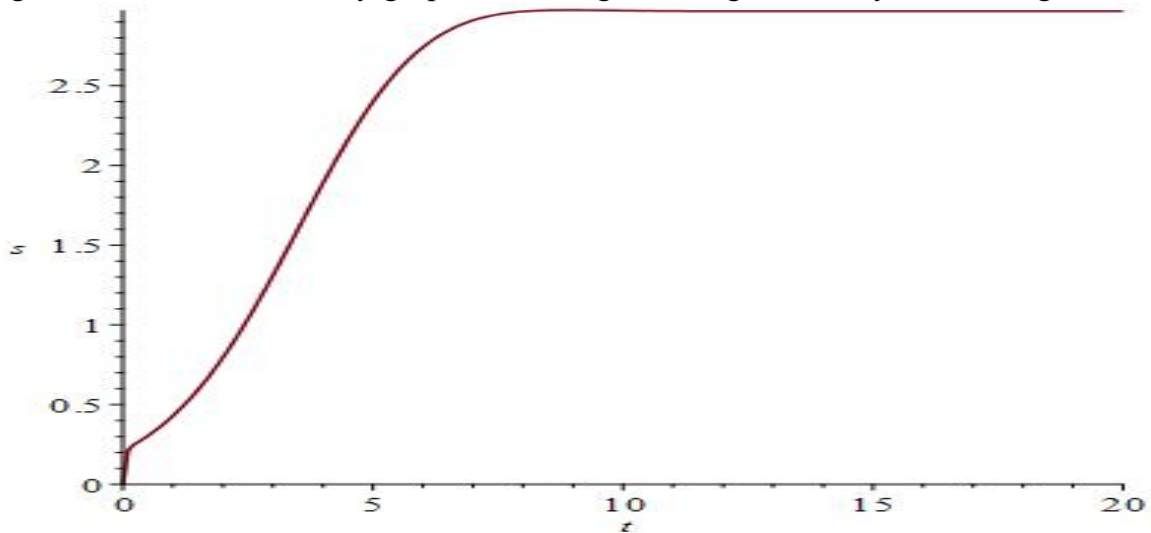

Figure 2 shows the susceptible among the resident of Ondo State

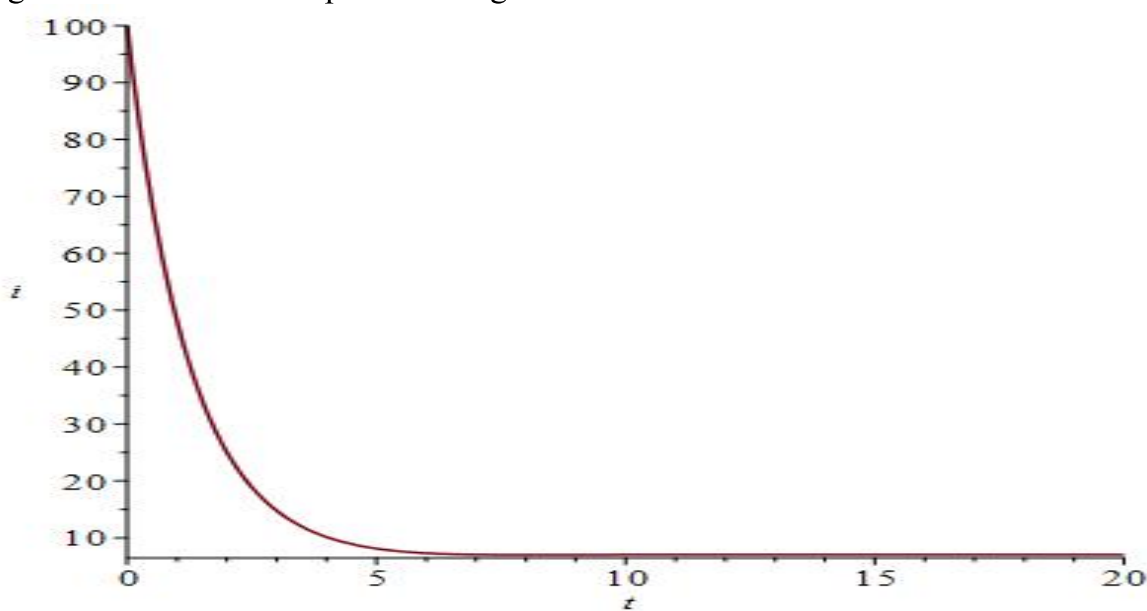

Figure 3 shows the infected among the resident of Ondo State 


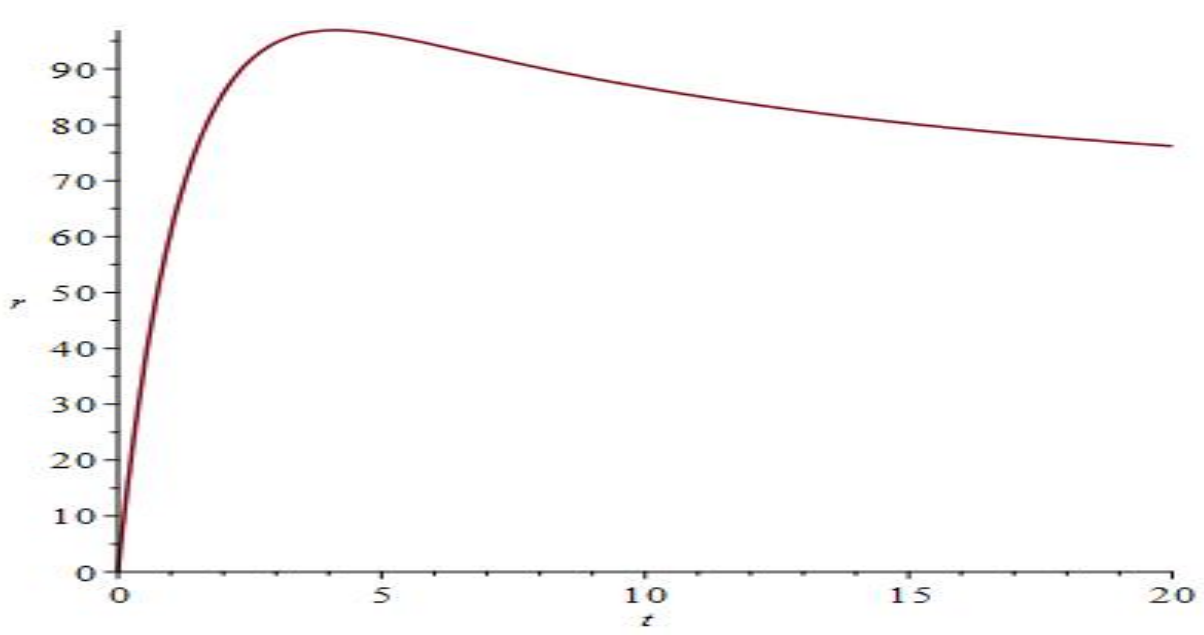

Figure 4 shows the recovered fromHBV among the resident of Ondo State

\section{Conclusion}

In this paper, we used a well-known Mathematical model of Kermach and Mc kendricto describe the transmission dynamics of Hapatitis B in Ondo State Nigeria. The model virus free equilibrium and virusendemic equilibrium were obtained, likewise the reproductive number $\left(R_{0}\right)$ was also derived. The model dequation was solved numerically using Maple 16 RK4 solver and the solution indicated that with vaccination and awareness among the residents of Ondo State, the disease will be controlled and probably eradicated.

\section{Acknowledgement}

Researchers of this present study acknowledge and sincerely appreciate the academic developer, (TertiaryEducation Trust Fund (TETFund, Nigeria) for sponsoring this research via its Institution Based Research (IBR) Intervention. The researchers also acknowledge and appreciate the Management of the Federal Polytechnic, Ile-Oluji, Ondo State for its support. The management's efforts have created the enabling environment which culminated in the success of this research.

\section{References}

$\mathrm{Su}$, B., Shou, W., Dorman, K. S., \& Jones, ,D. (2009). Mathematical modeling of immune response in tissue. Comput. math. methods med, 10(1), 9-38.

Mn, ,., ,Su,Y., \&Kuang, Y. (2008). Analysis of basic model of virus infection with a application to HBV infection. Rocky mt.J.. Math. 38(5), $1573-1585$.

Meltem, C (2019). A mathematical model of hepatitis B transmission in Turkey .Com. Fac.Sci.Univ.

Ank. Ser .Al Math,stat.68 (2), 1586-1595.

Murray, J..D. (1993). Mathematical biology. Springer- Verlage. New York, 1993.

Ledder, G. (2013) Mathematics for the life science, calculus modelling probability and dynamic system, Springer, USA.

KermackW.O and Mc Kendrick A.G (1927). Contribution to mathematical theory of epidemics, Soc. Lond .A mat..115,700-721.

James, D. S and Sleemen, D.D.(2003). Differential equations and mathematical biology .chapman and hall/CRC, London.

Chao, J., Chang, E.T. \& So, S.K.S. (2010). Hepatitis B and practices among healthcare and public health professionals in China: a cross-sectional study. BioMed Central Public Health, 10(98), 1-11. doi:10.1186/1471-2458-10-98

Codex. (2013). Forskningsominvolverarm änniskan - informeratsamtycke. Uppsala: Centrum förforsknings\&bioetik. Collected 26th of January, 2013, from http://www.codex.uu.se/manniska2.shtml

Dunford, L., Carr, M.J., Dean, J., Nguyen, L.T., Ta, T.T.H., Nguyen, B.T., Connell, J., Coughlan, S., Nguyen, H.T., Hall, W.W. \& Nguyen, T.L.A. (2012) A multicentre molecular analysis of hepatitis B and bloodborne virus coinfections in Viet Nam. PLoS ONE, 7(6). doi:10.1371/journal.pone.0039027

Duong, T.H., Nguyen, P.H., Henley. K. \& Peters, M. (2009). Risk factors for hepatitis B infection in rural Vietnam. Asian Pacific Journal of Cancer Prevention, 10(1), 97-102.

Glanz, K., Rimer, B.K. \& Viswanath, K. (red.) (2008). Health behavior and health education: theory, research and practice. (4. ed.) San Francisco: Jossey-Bass.

Hipgrave, D., Van, N., Huong, V., Long, H., Dat, D., Trung, T., Jolley, D., Maynard, J. \& Biggs, B-A. (2003). 
Hepatitis B infection in rural Vietnam and the implications for a national program of infant immunization. American Journal of Tropical Medicine and Hygiene, 69(3), 288-294.

Hwang, J., Huang, C-H. \& Yi, J. (2010). Knowledge about hepatitis B and predictors of hepatitis B vaccination among Vietnamese American college students. Journal of American College Health, 56(4), 377-382. doi:10.3200/JACH.56.44.377-382.

Lee, H-O., Lee, O-J., Kim, S., Hontz, I. \& Warner, A. (2007). Differences in knowledge of hepatitis B among Korean immigrants in two cities in the rocky mountain region. Asian Nursing Research, 1(3), 165-175. doi:10.1016/S1976-1317(08)60019-5

Ma, G.X., Shive, S.E., Fang, C.Y., Feng, Z., Parameswaran, L., Pham, A. \&Khanh, C. (2007). Knowledge, attitudes, and behaviors of hepatitis B screening and vaccination and liver cancer risks among Vietnamese Americans. Journal of Health Care for the Poor and Underserved, 18(1), 62-73. doi:10.1353/hpu.2007.0013

Nguyen, V.T.T., Law, M.G. \& Dore, G.J. (2008). An enormous hepatitis B virus-related liver disease burden projected in Vietnam by 2025. Liver International, 28(4), 525-531. doi:10.1111/j.1478-3231.2007.01646.x

Nguyen, V.T.T., McLaws, M-L. \& Dore, G.J. (2007). Highly endemic hepatitis B infection in rural Vietnam. Journal of Gastroenterology and Hepatology, 22(12), 2093-2100. doi:10.1111/j.1440-1746.2007.05010.x

Shepard, C., Simard, E., Finelli, L., Fiore, A. \& Bell, B. (2006). Hepatitis B virus infection: epidemiology and vaccination. Epidemiologic Reviews, 28(1), 112-125. doi:10.1093/epirev/mxj009

Socialstyrelsen. (2008). Vaccination av barn: Det svenskavaccinationsprogrammet. Enkunskaps översiktförh älsovårdspersonal. Socialstyrelsen. Collected 2th of April, 2013, from http://www.socialstyrelsen.se/Lists/Artikelkatalog/Attachments/8825/2008-126-9_20081269.pdf

Taylor, V.M., Choe, J.H., Yasui, Y., Li, L., Burke, N. \& Jackson, J.C. (2005). Hepatitis B awareness, testing, and knowledge among Vietnamese American men and women. Journal of Community Health, 30(6), 477490.

Vu, L., Gu, Z., Walton, J., Peet, A., Dean, J., Dunne, M. \&Debattista, J. (2012). Hepatitis B knowledge, testing, and vaccination among Chinese and Vietnamese adults in Australia. Asian-Pacific Journal of Public Health, 24(2), 374-384. doi:10.1177/1010539510390205

Weinbaum, C.M., Mast, E.E. \& Ward, J.W. (2009). Recommendations for identification and public health management of persons with chronic hepatitis B virus infection. Hepatology, 49(5), 35-44. doi:10.1002/hep.22882 